\title{
Applications of genomic medicine in endocrinology and post-genomic endocrine research
}

\author{
Constantine A. Stratakis
}

Heritable Disorders Branch, and the Section on Endocrinology \& Genetics, Developmental Endocrinology Branch, (DEB), National Institute of Child Health and Human Development (NICHD), National Institutes of Health (NIH), Bethesda, MD 20892, USA

\begin{abstract}
In the mid 1980's, two advances revolutionized Medicine in a way that is comparable only to some of the most important events in the approximately 3,000 years of its history. The first was the introduction of the concept of "positional cloning", i.e. the idea that one can identify genes for human disease though knowing nothing or very little about their function. The second was the discovery of the method of polymerase chain reaction (PCR) which made DNA easier to work with for all biomedical researchers and clinicians. Fresh in the history of Endocrinology were the great discoveries of neuroendocrinology, and even more contemporary and potent, the influence of the then emerging field of molecular endocrinology. Cancer medicine and traditional human genetics were the fields that benefited most from the first applications of the new genomic concepts and technologies. Almost two decades later, and after the first successful applications of positional cloning in Endocrine Genetics with the identification of RET, menin, PTEN and PRKARIA in the various forms of multiple endocrine tumor syndromes, and a number of other genes in developmental diseases affecting the pituitary, thyroid, parathyroid, pancreas, adrenal and gonadal glands, endocrinology has made a comeback to the forefront of "genomically"- influenced as well as post-genomic Medicine. This report, using the example of endocrine tumor genetics, presents the process and some of the accomplishments of positional cloning and discusses the influence of endocrinology on contemporary translational research. The author suggests that some of the most traditional endocrine concepts, established in the previous two centuries, could help us understand the complex pathways recently unraveled in cancer genetics and, consequently, other fields. It is suggested that "Endocrine" genes that control cellular signaling act as "conductors" since they regulate differentiation, growth and proliferation. Their complex function and resultant "transcriptomes" are now being investigated by post-genomic Medicine. In cancer research, endocrine genes defy classic definitions of tumor suppressors and oncogenes and regulate gatekeepers, caretakers, and landscapers. In the post-genomic, translational Medicine, Endocrinology once again could help us to understand cellular regulation and pathophysiology and to design new treatments.
\end{abstract}

Key words: Cancer, Genetics, Cloning, Tumor, Suppressor, Gene, Oncogene, Gatekeeper 


\section{INTRODUCTION}

Medicine is an ever changing art, continuously adjusting to the constant discoveries of science. This was admirably expressed by Hippocrates: Change in Medicine, is profound and contrasts remarkably with the immutable nature of other Sciences. "Medicine cannot be learned quickly because it is impossible to create any established principle in it, the way that a person who learns writing according to one system that people teach understands everything; for all who understand writing in the same way do so because the same symbol does not sometimes become opposite, but is always steadfastly the same and not subject to chance. Medicine, on the other hand, does not do the same thing at this moment and the next, and it does opposite things to the same person, and often things that are self-contradictory". ${ }^{1}$

Nowhere is more evident the continuous shifting of ideas and practices in modern Medicine than in cancer genetics, a field that stands at the forefront of genomic medicine. Evolution is so fast that, to most observers, the core of contemporary discoveries and resultant theories is not visible. There does not appear to be a theme, and when a model is proposed, it quickly disintegrates and gets buried under contradictory evidence and new data. A perfect example is that of the concept of "landscaper" genes that was introduced and supported by a few $^{2}$ to be recently questioned by many.,4

Does this observation validate what was earlier mentioned? Is there, in Medicine, truly a "constant rebirth of the same old principles with a new face"? For an endocrinologist, this hypothesis could not even be questioned; for endocrinologists are used to change, as long as the basic principle of cellular communication is respected. Endocrinology is, after all, "the study of communication and control within a living organism by means of chemical messengers that are synthesized in whole or in part by that organism".

The basic thesis of this article is that Endocrinology is uniquely positioned in the post-genomic era of Medicine. After reviewing the basic principles of positional genetics, the author uses his experience from the field of cancer genetics to suggest that endocrinologists are poised to become very familiar with translational medicine, as the many interactions of some classic - and a few novel - "endocrine" genes are unraveled by expression profiling and other techniques. It is along these lines that these genes are called "conductors", to contrast them with the "gatekeepers", "caretakers" and "landscapers" of contemporary cancer research. Similar endocrine concepts may help shape the understanding of new discoveries in other fields of Medicine in a way similar to how classic Endocrinology assisted in the understanding of the great discoveries of neurobiology and immunology in previous decades.

\section{GENOMIC ENDOCRINOLOGY: POSITIONAL CLONING OF ENDOCRINE GENES}

\section{The principles of positional genetics}

In the process of disease gene identification, the candidate gene approach relies on partial knowledge of the disease. This process was successful in identifying disease genes whose function was obvious. For example, the genetic defects of most of the hereditary enzymatic disorders, including congenital adrenal hyperplasia (CAH) syndromes, became known in the late '80s, when the introduction of polymerase chain reaction (PCR) made the tools of molecular biology widely available to the medical and genetic research community. However, at about the same time, research stumbled on diseases without any obvious candidate genes (e.g. the multiple endocrine neoplasia (MEN) syndromes), or diseases that the screening of obvious candidate genes frequently failed to reveal mutations (for example, familial hypoparathyroidism, in the majority of cases, is not caused by mutations in the parathyroid hormone gene). It was in these diseases that "reverse genetics", or more appropriately "positional cloning", ${ }^{6}$ offered a solution. Positional cloning is complemented by the Human Genome Project (HGP) and the world wide web in making available, in a fast and controlled manner, information that would otherwise be inaccessible. ${ }^{7}$

The process of positional genetics is outlined in figure 1 . The first step is the collection of clinical information from families with affected members, 


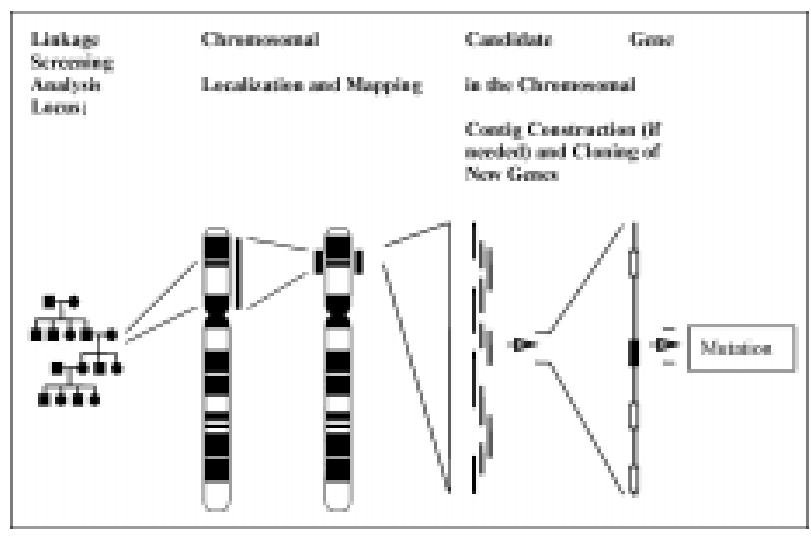

Figure 1. The steps of positional cloning (see text).

the determination of the mode of inheritance of the defect (autosomal dominant or recessive, X-linked, complex inheritance), and the phenotyping of subjects (or tissues) following well-established criteria for the diagnosis of the disorder under investigation. If inheritance is not known, formal segregation analysis needs to be performed to determine the autosomal or X-linked, and the recessive or dominant nature of the inheritance. ${ }^{8}$ Once this is determined and the penetrance of the disorder is known, appropriate linkage software may be used. ${ }^{9}$ For more information on currently available software packages, the reader may check http://darwin.case.edu and other related links.

Linkage is examined with polymorphic markers that cover the entire human genome; ${ }^{10}$ any marker that shows polymorphism and is known to lie close to or within a putative disease gene may be used. Genetic linkage can be defined as the tendency for alleles in close proximity on the same chromosome to be transmitted together as an intact unit during meiosis. The strength of linkage can then be used as a unit of measurement to find out how close genetically different loci are to each other. This unit of map distance is an approximation of physical distance but is also highly dependent on other factors (for example, the frequency of recombination is not the same in both genders, differing along the length of chromosomes and through the various chromosomes). The likelihood (Logarithm of Odds or LOD score) method is the most widely used for linkage analysis.
Once a locus on a chromosome has been identified, narrowing of the region (this region is usually several thousands of base-pairs-long) is done through the analysis of informative recombinations in the studied patients. The disease region may harbor already mapped genes. On-line databases, (Gen Bank, ENSEMBL - www.ensembl.org, and others, and especially for clinicians the "on line Mendelian inheritance in man (OMIM)", ${ }^{11}$ may provide all the necessary information. If a transcript is a reasonable candidate, mutation screening may identify the disease gene. If, however, these steps fail to identify the disease gene, cloning of new sequences from the area may be needed. This is usually done through the construction of a "contig", a contiguous array of human DNA clones covering the disease region. These DNA clones are maintained in large vectors like bacterial artificial chromosomes (BACs); P1 and cosmid clones may also be used for coverage of the entire region or a subset of it. Contig construction may take a long time depending on the genetic distance to be covered and the features of the genomic region. The identification of new genes and the construction of the contig is also called "chromosomal walking". The building blocks of this "walk" are the individual clones, which are linked together by sequence-tagged sites (STSs) that are present in more than one clone, thus providing their proper order. Polymorphic markers (including those that were used for the linkage part of the process) are the most useful STSs because they provide a direct link between the genetic and the physical mapping data. Individual clones can be sequenced; genes are identified in this process through their unique sequence features or through in vitro translation. The latter usually provides expressed sequence tags (ESTs) which can then be analyzed through software that is available on line to determine the full gene structure and identify redundancies (multiple ESTs or STSs of the same gene) and other errors. Each one of the newly identified genes may be screened for mutations, as long as the expression profile of the identified transcript matches the spectrum of pathology of the tissues affected by the disease under investigation. Although this is helpful for most diseases, for others the expression profile may even be misleading; thus, the presence of a transcript in an 
affected tissue does not always match the phenotype. Complete segregation of the disease with an identified mutation, functional proof, and/or mutations in two or more families with the same disease are usually needed as supportive evidence that the cloned sequence is the disease gene. ${ }^{12}$

\section{Positional cloning of "endocrine" genes}

A number of genes were identified by positional cloning in endocrinology in the 90's. It is worth noting, however, that endocrine tumor syndromes, despite their rarity and small overall impact on everyday clinical endocrine practice, represent a disproportionately large group of diseases molecularly elucidated by positional cloning.

There are, most likely, two reasons for this phenomenon. First, for most endocrine tumor syndromes there were no obvious candidates, unlike, for example, conditions such as CAH. Second, positional cloning of genes responsible for familial tumor syndromes is greatly assisted by the use of neoplastic tissue for studies such as loss-of-heterozygosity (LOH), comparative genomic hybridization $(\mathrm{CGH})$ and other fluoroscent in situ hybridization (FISH) applications. These techniques narrow the genetically defined chromosomal regions and may with precision identify the responsible genes; $\mathrm{LOH}$ studies were critical in the identification of VonHippel-Lindau disease (VHL-elongin), ${ }^{13} \mathrm{MEN} 1$ (menin),${ }^{14}$ Cowden disease (PTEN), ${ }^{15}$ Peutz-Jeghers syndrome $(S T K 11 / L K B 1)^{16,17}$ and Carney complex $(P R K A R 1 A)^{18}$ genes.

\section{ENDOCRINE TUMOR SUPPRESSOR GENES: THE CONCEPT OF "CONDUCTORS"}

The genes that have been identified by positional cloning in endocrine tumor genetics are unique in that, although most appear to function as classic tumor suppressors (with the notable exception of RET in the MEN 2 syndromes $^{19}$ ), no one functions as a direct controller of the cell cycle or apoptosis. Thus, these genes cannot be characterized as "gatekeepers" ${ }^{20}$ Nor can they be defined as "caretakers", since none of the endocrine tumor genes participates in the maintenance of chromosomal or nucleotide stability. Therefore, what best characterizes endocrine tumor genes?
Most endocrine human genes participate in complex and highly interactive signaling pathways; they not only control a number of other genes but they also affect communication of multiple pathways. As noted elsewhere ${ }^{21}$ this is reminiscent of the internet, where the relevant genes may be "nodes" or "hubs" in this internet-like structure. ${ }^{22}$ As it happens, most "endocrine" genes constitute parts of important cellular "hubs"!

Can endocrine genes be functioning as classic tumor suppressor genes? For some of these genes the answer is unequivocally "yes": both VHL and menin act as classic tumor suppressor genes. ${ }^{23,24} \mathrm{VHL}$ may also be categorized as a "gatekeeper". ${ }^{20}$ For other endocrine tumor genes, a different term has been proposed, namely "landscapers". ${ }^{20}$ It appears that PTEN, a protein tyrosine phosphatase first found mutated in Cowden disease ${ }^{15}$ and subsequently in numerous sporadic neoplasms, including thyroid cancer, ${ }^{25}$ fits the definition of a "landscaper": a protein that is mostly (or, perhaps, exclusively) present in the cytoplasm, where it interacts with a variety of molecules, participating in several pathways and affecting stromal-epithelial interactions, creating an abnormal microenvironment that eventually leads to epithelial cell transformation and tumor formation through complex interactions with proteins as distinct as bone morphogenetic proteins (BMPs) and others. ${ }^{26}$

Nevertheless, if PTEN is a "landscaper", what is the designation of the last in the series of endocrine tumor genes to be positionally cloned, i.e. PRKAR1A, the Carney complex gene ${ }^{18}$ Even more clearly than PTEN, PRKAR1A controls a hormonal signaling pathway that is seminal for endocrine function; it controls growth and proliferation signals and has a great number of interactions with a variety of other signaling systems. ${ }^{27} P R K A R 1 A$ is an example of an endocrine gene, positionally identified as a tumor suppressor, which may be called a "conductor"; a controller of a hub of cellular functions, expressed in all cells and presenting multiple faces, and sensitively adapting each time to cellular needs. ${ }^{28}$

Another example of a "conductor" endocrine gene whose role in cancer research defies the classic definitions of a tumor suppressor or an onco- 
gene is that of TGF-b..$^{29}$ A variety of endocrine genes, some with diverse developmental roles, like BMPs and their receptors, as well as menin and PTEN through their interactions with the Smad family of proteins, participate in the complex TGF-b signaling cascade. ${ }^{30}$ Most of the genes responsible for a number of human diseases, including endocrine tumor syndromes, were positionally cloned. ${ }^{26}$

\section{POST-GENOMIC ENDOCRINOLOGY}

In the history of cancer genetic research, the direction of positional identification of the various classes of genes was the reverse of that of the basic theorem of tumor development (Figure 2). The concept of "genes-conductors" is a direct evolution of that of a "landscaper", and it is endocrinology's contribution to genomic Medicine's great discoveries in cancer research.

It was recently stated that "progress in dissecting signaling pathways has begun to lay out a circuitry that will likely mimic electronic integrated circuits in complexity and finesse, where transistors are

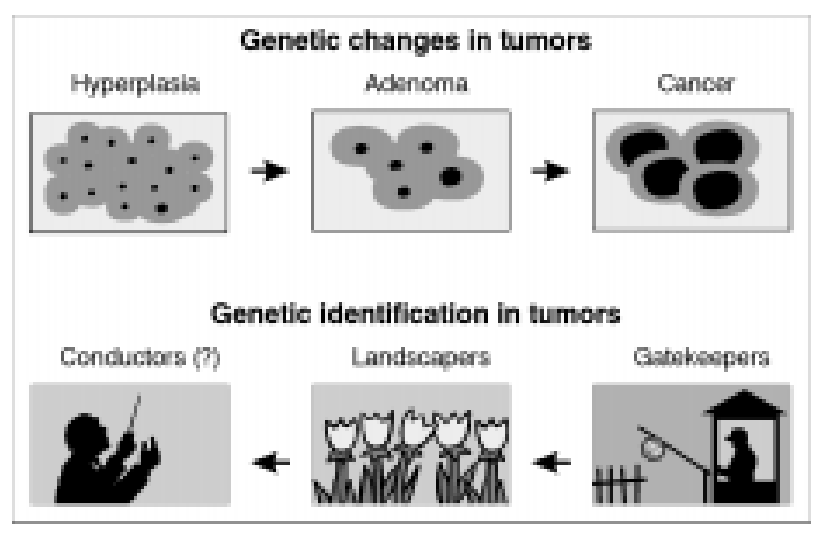

Figure 2. Historically, gene identification in cancer research followed the reverse order of that suggested in the main theorem of tumor development: benign hyperplasia is more than likely caused by a small number of changes in central, "conductor" genes that are only now being discovered, and growth to an adenoma involves the complex interplay of "landscapers" (as well as "caretakers"), genes that act mostly in a tumor suppressor function and were identified in the mid-90's; finally, cancer and metastasis largely depend on the impaired function once more of "gatekeepers", genes that directly control the cell cycle, almost all being tumor suppressors that were first discovered in the late ' 80 's- early '90's. replaced by proteins (e.g. kinases and phosphatases) and the electrons by phosphates and lipids". ${ }^{31}$ Although this statement is related to cancer research ${ }^{31}$ translational Medicine today identifies these complex interactions everywhere, ${ }^{32}$ making it no surprise that $30,000-40,000$ genes (rather than 100,000 genes $^{33}$ are sufficient to produce the human phenotype. It seems that it is not the number of genes that is important but rather the number and quality of interactions between them, as well as their products and translational regulation.

An almost perfect example of an endocrine conductor transcriptome is the one recently presented by Roberts et $\mathrm{al}^{34}$ : the description of mitogen-activated protein kinase (MAPK) pathways during yeast pheromone response. Similar analyses are currently being used in many experiments, not only in endocrine tumors but also in a variety of other applications. ${ }^{35}$ In this process, investigations by microarrays, collection of probes for hundreds of genes at a time (or the entire genome, depending on the type of the array used) have become a standard. An example from a recent study is shown in figure $3 .^{36}$

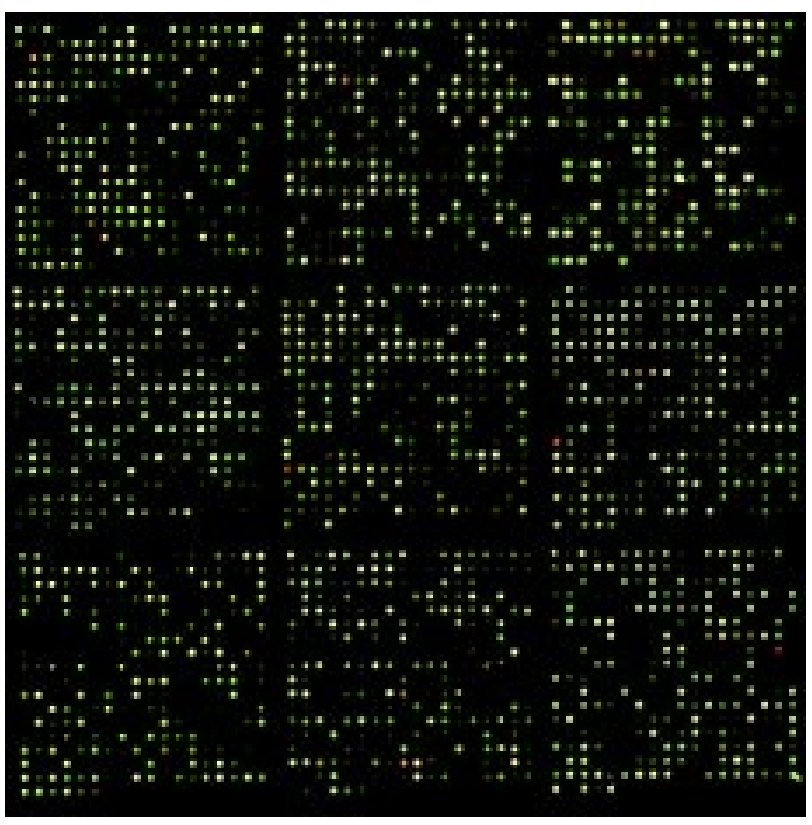

Figure 3. A cDNA array: fluorescent labeled cDNA probes, $\mathrm{ACTH}$-independent bilateral macronodular adrenal hyperplasia (Cye 3) as compared to ACTH-dependent hyperplasia (Cye5), were hybridized to glass slides containing genes involved in oncogenesis. 
Hence, the basic theme of this article can be summarized as follows: In post-genomic Medicine, the investigation of the transcriptome by microarrays (Figure 3) and other means offers a new opportunity for endocrinologists to actively participate in biomedical research. Endocrine concepts and the reemergence of signaling as a primary means of understanding the complex interactions of genes may shape the new bioinformatics tools that are needed for the analysis of pertiment data, as well as direct the development of new drugs that are tailored to genomic-translational information. ${ }^{37,38}$

\section{REFERENCES}

1. Potter P, 1995 Hippocrates "Places in man". Harvard University Press, Cambridge; p, 81.

2. Kinzler KW, Vogelstein B, 1998 Landscaping the cancer terrain. Science 280: 1036-1037.

3. Woodford-Richens K, Williamson J, Bevan S, et al, 2000 Allelic loss at SMAD4 in polyps from juvenile polyposis patients and use of fluorescence in situ hybridization to demonstrate clonal origin of the epithelium. Cancer Res 60: 2477-2482.

4. Playford RJ, 2001 Landscaper seeks remunerative position Gut 48: 594-595.

5. Becker KL, Nylen ES, Snider RH, 1995 Endocrinology and the endocrine patient. In Becker KL et al (eds) Principles and practice of Endocrinology and metabolism. J.B. Lippincott; pp, 2-8.

6. Collins FS, 1992 Positional cloning: let's not call it reverse anymore. Nature Genet 1: 3-6.

7. Borsani G, Ballabio A, Banfi S, 1998 A practical guide to orient yourself in the labyrinth of genome databases. Hum Mol Genet 7: 1641-1648.

8. Khoury MJ, Beaty TH, Cohen BH, 1993 Fundamentals of genetic epidemiology. Oxford University Press.

9. Terwillinger JD, Ott J, 1994 Handbook of human genetic linkage. The Johns Hopkins University Press.

10. Collins FS, 1995 Positional cloning moves from perditional to traditional. Nature Genet 9: 347-350.

11. Online Mendelian Inheritance in Man, OMIM, 1998 Center for Medical Genetics, Johns Hopkins University and National Center for Biotechnology Information, National Library of Medicine.

12. Bishop MJ, 1997 Gene mapping and isolation. Access to databases. Methods Mol Biol 68: 237-259.

13. Linehan WM, Lerman MI, Zbar B, 1995 Identification of the von Hippel-Lindau (VHL) gene. JAMA 273: 564-570.

14. Liaw D, Marsh DJ, Li J, et al, 1997 Germline mutations of the PTEN gene in Cowden disease, an inherited breast and thyroid cancer syndrome. Nat Genet
16: 64-67.

15. Chandrasekharappa SC, Guru SC, Manickam P, et al, 1997 Positional cloning of the gene for multiple endocrine neoplasia-type 1 . Science 276: 404-407.

16. Hemminki A, Markie D, Tomlinson I, et al, 1998 A serine/threonine kinase gene defective in Peutz-Jeghers syndrome. Nature 391: 184-187.

17. Jenne DE, Reimann H, Nezu J, et al, 1998 Peutz-Jeghers syndrome is caused by mutations in a novel serine threonine kinase. Nat Genet 18: 38-43.

18. Kirschner LS, Carney JA, Pack SD, et al, 2000 Mutations of the gene encoding the protein kinase A type Ialpha regulatory subunit in patients with the Carney complex. Nat Genet 26: 89-92.

19. Eng C, 1996 The RET proto-oncogene in Multiple Endocrine Neoplasia type 2 and Hirschprung's disease. N Engl J Med 335: 943-951.

20. Macleod K, 2000 Tumor suppressor genes. Curr Op Genet Dev 10: 81-93.

21. Vogelstein B, 2000 Complexity in cancer. Nature 408: 307-311.

22. Barabasi AL, Oltvai ZN, 2004 Network biology: understanding the cell's functional organization. Nat Rev Genet. 5: 101-113.

23. Iliopoulos O, 2001 von Hippel-Lindau disease: genetic and clinical observations Front Horm Res 28: 131166.

24. Chandrasekharappa SC, Teh BT, 2001 Clinical and molecular aspects of multiple endocrine neoplasia type 1 . Front Horm Res 28: 50-80.

25. Yamada KM, Araki M, 2001 Tumor suppressor PTEN: modulator of cell signaling, growth, migration, and apoptosis. J Cell Sci 114: 2375-2382.

26. Eng C, 2001 To be or not to BMP. Nat Genet 28: 105107.

27. Stratakis CA, Cho-Chung YS, 2002 Protein kinase A and human disease. Trends Endocrinol Metab 13: 5052.

28. Stratakis CA, 2003 Genetics of adrenocortical tumors: gatekeepers, landscapers and conductors in symphony. Trends Endocrinol Metab 14: 404-410.

29. Derynck R, Akhurst RJ, Balmain A, 2001 TGF-b signaling in tumor suppression and cancer progression. Nat Genet 29: 117-129.

30. Massaguı J, Blain SW, Lo RS, 2000 TGF-b signaling in growth control, cancer, and heritable disorders. Cell 103: 295-309.

31. Hanahan D, Weinberg RA, 2000 The hallmarks of cancer. Cell 100: 57-70.

32. Hillan KJ, Quirke P. Preface to genomic pathology. J Pathol 195: 1-2.

33. The Genome International Sequencing Consortium, 2001 Initial sequencing and analysis of the human genome. Nature 409: 860-921.

34. Roberts CJ, Nelson B, Marton MJ, et al, 2000 Signaling and circuitry of multiple MAPK pathways revealed 
by a matrix of global gene expression profiles. Science 287: 873-880.

35. Alizadeh AA, Ross DT, Perou CM, van de Rijn M, 2001 Towards a novel classification of human malignancies based on gene expression patterns. J Pathol 195: 41-52.

36. Bourdeau I, Antonini SR, Lacroix A, et al, 2004 Gene array analysis of macronodular adrenal hyperplasia confirms clinical heterogeneity and identifies several candidate genes as molecular mediators. Oncogene 23:
1575-1585.

37. Marton MJ, DeRisi JL, Bennett HA, et al, 1998 Drug target validation and identification of secondary drug target effects using DNA microarrays. Nat Med 4: 12931301.

38. Khan J, Wei JS, Ringner M, et al, 2001 Classification and diagnostic prediction of cancers using gene expression profiling and artificial neural networks. Nat Med 7: 673-679. 